\title{
The value of a thorough protocol in the investigation of sudden infant deaths
}

\author{
David W Sadler
}

\begin{abstract}
Aims-To review the diagnostic value of using a thorough necropsy protocol for the investigation of sudden infant deaths, with particular emphasis on the value of routine ancillary laboratory investigations. Methods-The necropsy and related records of all neonatal, infant, and young childhood deaths (under three years) referred for medicolegal investigation at Dundee from 1990 to early 1998 were reviewed retrospectively. Relevant positive and negative findings were abstracted from the police reports, hospital medical records, necropsy reports, and the results of routine bacteriological, virological, toxicological, and biochemical laboratory investigations.
\end{abstract}

Results-Within the study period, 63 deaths presented as apparent "cot deaths," nine as suspected homicides, nine as neonatal deaths, and 14 in some other manner. An adequate cause of death was identified on the basis of necropsy and laboratory investigations in $35 \%$ of the 63 apparent cot deaths, leaving $63 \%$ to be finally categorised as sudden infant death syndrome (SIDS). Ten (16\%) of the apparent cot deaths were explained on the sole basis of unexpected positive microbiological findings, mostly pneumococcal or meningococcal meningitis and/or septicaemia. Petechial haemorrhages were identified at one or more intrathoracic site in $90 \%$ of SIDS and in $55 \%$ of explained cot deaths. Conclusions-Early and extensive laboratory investigations performed routinely in apparent cot deaths provide an unexpectedly high positive diagnostic yield. Routine early bacteriological culture of the CSF (by cisternal puncture) and blood before necropsy should be mandatory in the investigation of all sudden infant deaths. (F Clin Pathol 1998;51:689-694)

University Department of Forensic Medicine, Dundee Royal Infirmary, Dundee, UK

D W Sadler

Correspondence to: Dr David W Sadler, University Department of Forensic Medicine, Dundee Royal Infirmary, Dundee DD1 9ND, Scotland, UK; email:

d.w.sadler@dundee.ac.uk

Accepted for publication 13 May 1998

originally $c$ death of any infant or young child, which is unexpected by history, and in which a thorough postmortem examination fails to demonstrate an adequate cause of death." ${ }^{1}$ This definition was endorsed at the second SIDS global strategy meeting in Norway in $1994 .^{2}$ However, this definition requires further scrutiny since it does not state how "sudden" the death must be, to whom it is "unexpected," what constitutes a "thorough" necropsy, or what constitutes an "adequate cause of death." ${ }^{3}$ Although SIDS is a valuable term it also contributes to negative expectations in relation to infant deaths and may allow convenient categorisation without adequate investigation. Since SIDS is a diagnosis by exclusion, geographical differences in its incidence will depend on how thoroughly infant deaths are investigated in each area. Accordingly, "SIDS" appears as the final diagnosis following $50-90 \%$ of infant necropsies. ${ }^{4}$

The infant necropsy protocol used in Dundee since 1990 exceeds the recommendations of the Royal College of Pathologists ${ }^{5}$ and includes numerous routine bacteriological, virological, biochemical, cytogenetic, and radiological investigations, in addition to the usual thorough necropsy and histological examinations recommended in most infant necropsy protocols. ${ }^{36}$ Since 1991, arrangements have existed in Tayside for the police and procurators fiscal to call out a forensic pathologist so that infant necropsies can start within a few hours of death. The median time between death and necropsy has been reduced from 27 hours in 1990 (mean 35 hours, range 2.0 to 120 hours) to five hours in subsequent years (mean 11 hours, range 2.5 to 53 hours). As a result, one would expect the incidence of postmortem microbiological, biochemical, and autolytic artefacts to be reduced. Experience of this infant death protocol is reviewed here, with particular emphasis on the diagnostic value of routine microbiology and other ancillary laboratory investigations.

\section{Methods}

The necropsy and related records of all neonatal, infant, and young childhood deaths (under three years) referred for medicolegal investigation to the procurators fiscal of Tayside and north-east Fife from 1990 to early 1998 were reviewed retrospectively. The majority of these cases presented as "cot deaths." Relevant positive and negative findings were abstracted from the police reports, hospital medical records, necropsy reports, and the results of further laboratory investigations. Histological examinations were in accordance with the recommendations of the Royal College of Pathologists $^{6}$ but routine neuropathological examinations were not performed. The full necropsy and laboratory investigation protocol is given in the appendix.

\section{Results}

There were 95 deaths during the study period: 63 presenting as apparent cot deaths, nine as suspected homicides, nine as neonatal deaths, and 14 in some other accidental or natural manner (fig 1). 
Of the 63 cases which presented as apparent cot deaths, 51 were provisionally diagnosed as SIDS after necropsy but only 40 of these were finally categorised as SIDS after exhaustive laboratory investigations. Two of the deaths attributed primarily to SIDS had other significant contributory factors listed in the cause of death - one with coxsackie B infection and one with hypohidrotic ectodermal dysplasia associated with early Klebsiella pneumoniae lung infection. In one case, which presented initially as an apparent cot death, overlaying remained a strong possibility and the cause of death was left undetermined.

Twenty two of the 63 apparent cot deaths $(35 \%)$ were satisfactorily explained by the identification of an adequate cause of death: six primarily on the basis of necropsy, five on histology, nine on bacteriology, one on virology, and one on biochemistry (fig 2). Necropsy revealed two cases of acute tracheobronchitis (both caused by $H$ influenzae), and one case each of acute epiglottitis ( $H$ influenzae), pneumonia (organism unknown), intussusception, and inhalation of vomit. All six cases were accompanied by confirmatory histological findings. Histology unexpectedly revealed three cases of pneumonia (one caused by $H$ influenzae and two with negative microbiology) and two cases of acute epiglottitis and pneumonia (one caused by mixed Staphylococcus aureus, group B streptococcus and adenovirus infection and one by mixed $H$ parainfluenzae and adenovirus infection). Ten deaths were explained solely on the basis of unexpected positive microbiological findings, accompanied by negative or minimal macroscopic and histological findings. These comprised two cases of pneumococcal meningitis and septicaemia, two cases of pneumococcal meningitis, two cases of meningococcal meningitis, and one each of group B streptococcal meningitis and septicaemia, Haemophilus influenzae pneumonia and septicaemia, Haemophilus influenzae meningitis, and coxsackie $B$ meningitis. Table 1 shows the yield of significant bacterial pathogens and commensals/contaminants at various sites.

Routine virological studies (serology, throat swab, ileal and faecal culture, and occasionally CSF culture) were performed in 58 cases, with

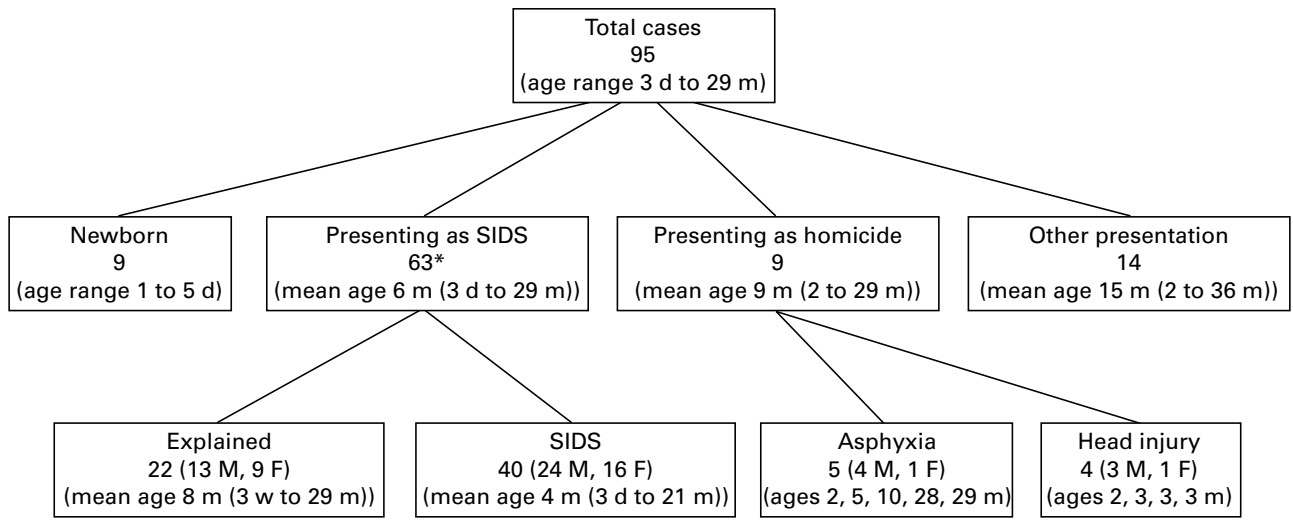

*Includes 17 month old in which cause of death was left undetermined

Figure 1 Breakdown of deaths in children under three years of age.

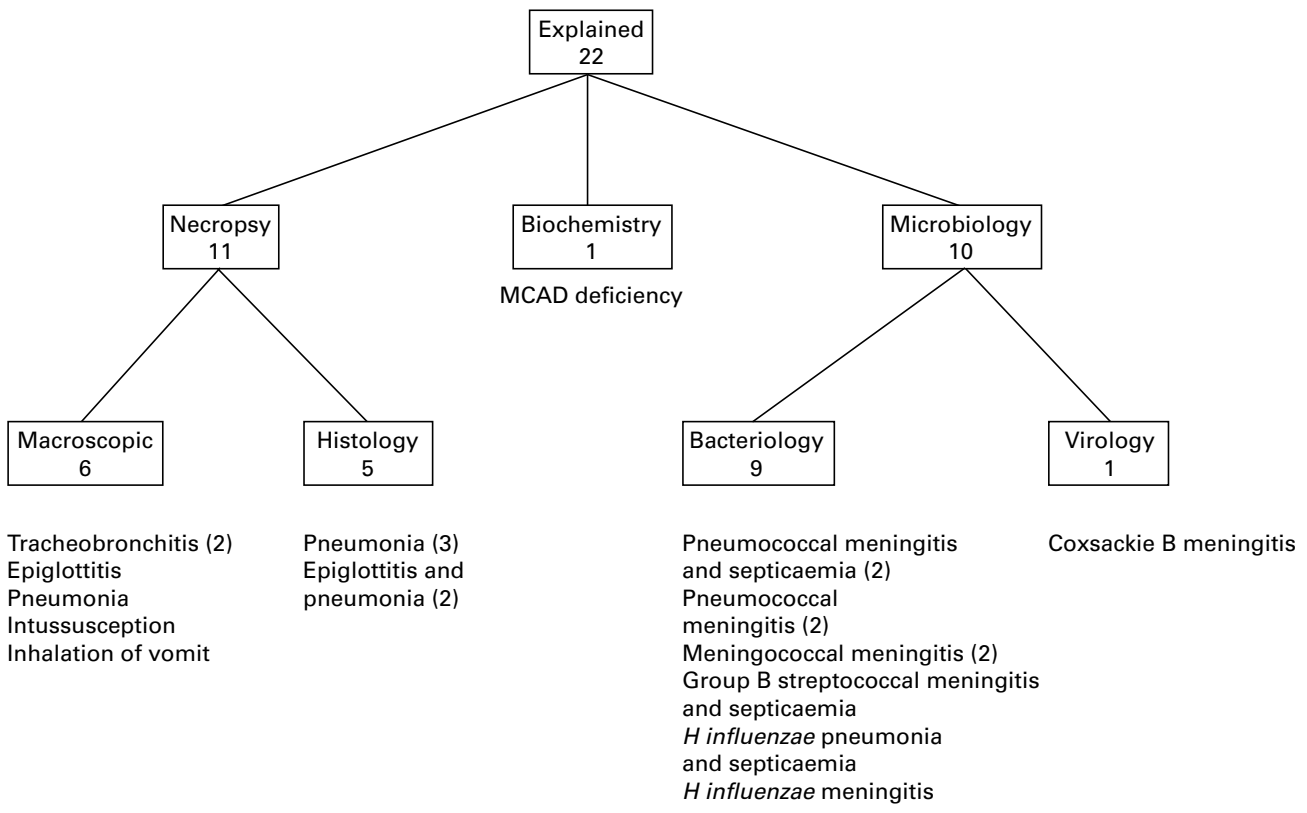

Figure 2 Cases presenting as cot deaths which were adequately explained. 
Table 1 Results of bacteriological investigations

\begin{tabular}{lllll}
\hline Site & Cultured & $\begin{array}{l}\text { No } \\
\text { growth }\end{array}$ & $\begin{array}{l}\text { Significant } \\
\text { growth }\end{array}$ & $\begin{array}{l}\text { Contaminant/ } \\
\text { commensal } \\
\text { growth }\end{array}$ \\
\hline CSF & 61 & 35 & $6^{\mathrm{a}}$ & $24^{\mathrm{b}}$ \\
Blood & 45 & 9 & $5^{\mathrm{c}}$ & $31^{\mathrm{d}}$ \\
Spleen & 21 & 13 & 0 & $8^{\mathrm{e}}$ \\
Middle ear & 15 & 5 & $2^{\mathrm{f}}$ & $8^{\mathrm{g}}$ \\
$\begin{array}{l}\text { Throat } \\
\text { Nose }\end{array}$ & 27 & 9 & $2^{\mathrm{h}}$ & $17^{\mathrm{i}}$ \\
$\begin{array}{l}\text { Trachea/ } \\
\text { bronchi }\end{array}$ & 19 & 6 & 0 & $11^{\mathrm{j}}$ \\
$\begin{array}{l}\text { Lung } \\
\text { Lung }\end{array}$ & 11 & 1 & $6^{\mathrm{k}}$ & $4^{\mathrm{l}}$ \\
\hline
\end{tabular}

a $S$ pneumoniae, Gram negative intracellular diplococci seen on $\mathrm{CSF}$ microscopy but not grown (2), $\mathrm{N}$ meningitidis grown, $H$ influenzae, group B streptococci. Another death attributed to pneumococcal meningitis had the organism in the middle ears but not in the CSF, where polymorphs were identified but only coliforms were grown.

${ }^{\mathrm{b}}$ Eight different organisms including $S$ epidermidis, coliforms, $S$ aureus, and various streptococci.

${ }^{c}$ H influenzae (2), $S$ pneumoniae (2) and group B streptococci.

${ }^{d}$ Seventeen different organisms including $S$ epidermidis, various streptococci and other bowel and skin flora.

${ }^{\mathrm{e}}$ Coliforms and bowel flora.

S pneumoniae.

${ }^{\mathrm{S}} \mathrm{S}$ pneumoniae, coliforms, H Influenzae.

${ }^{\text {h }} H$ parainfluenzae, group B streptococci.

Coliforms, $S$ aureus, $H$ influenzae and parainfluenzae, group B streptococci.

i $S$ aureus (9), S pyogenes, group B and other unspecified streptococci.

${ }^{\mathrm{k}} H$ influenzae (3), $H$ parainfluenzae, group B streptococci, $S$ Pneumoniae.

${ }^{1} S$ aureus (9), S pyogenes, H parainfluenzae, coliforms.

${ }^{\mathrm{m}}$ K pneumoniae.

${ }^{n} S$ aureus, $H$ influenzae, Klebsiella spp.

no significant growth in 40. Positive virology of major significance was in reported in three cases (coxsackie B meningitis, generalised coxsackie $\mathrm{B}$ infection involving the throat, lung, $\mathrm{CSF}$, and faeces, and adenovirus in the lung and throat). Less significant positive results, reported in 15 cases, comprised poliovirus in the throat or faeces (11), rotavirus (2), echovirus in the faeces, and influenza virus in the throat.

A 23 month old child, presenting as an apparent cot death, was provisionally diagnosed as Reye's syndrome after necropsy and histological examination. However, subsequent biochemical investigations revealed evidence of medium chain acyl coenzyme-A dehydrogenase (MCAD) deficiency and further cytogenetic investigations confirmed that she was homozygous for this autosomal recessive disorder.

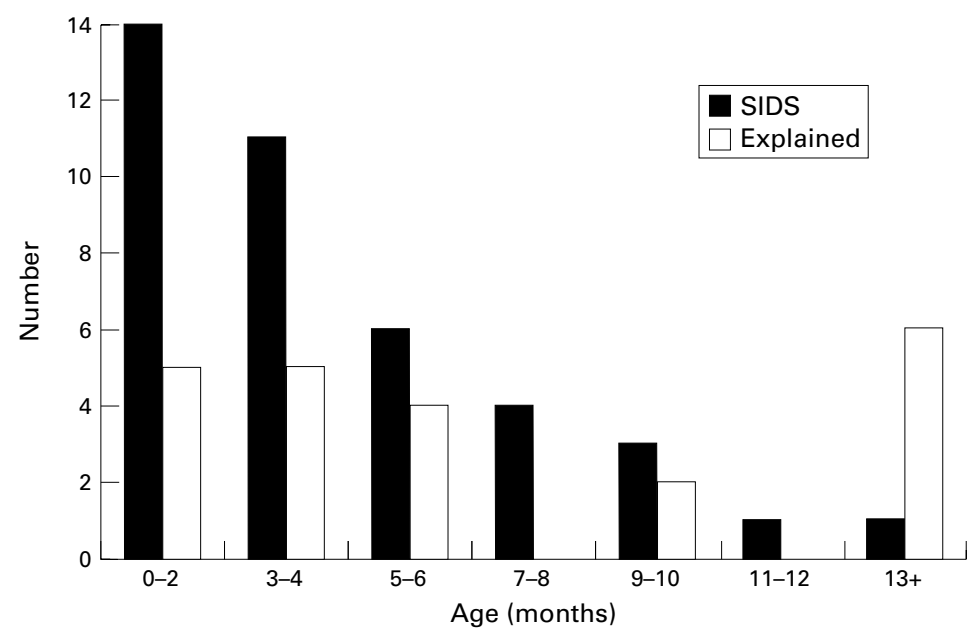

Figure 3 Age at death (SIDS cases and explained "cot death").
Figure 3 shows that, as expected, the incidence of SIDS was greatest in the first four months of life (mean 6.0 months, median 3.0 months) and declined with age, whereas apparent cot deaths which were ultimately explained had a more uniform age distribution (mean 8.0 months, median 5.5 months).

Petechial haemorrhages were present in at least one intrathoracic site in 36 of 40 SIDS cases $(90 \%)$ and in 12 of 22 explained infant deaths $(55 \%)$. The distribution of petechiae in both these groups is presented in table 2 . In the 40 cases of SIDS, petechial haemorrhages were absent in four cases and present at one, two, and all three intrathoracic sites in four, 14, and 18 cases, respectively. In the 22 explained infant deaths, petechial haemorrhages were absent in nine cases and present at one, two, and all three intrathoracic sites in four, six, and two cases, respectively; the remaining case-a 17 month old boy who died from inhalation of vomit-had external petechiae over the face but none internally.

Potentially important circumstantial evidence was mentioned in the police reports in 18 of the 40 cases finally categorised as SIDS. Six infants were found dead in bed with their parents and one lying prone on its sleeping father's chest. Two infants were described as being overwrapped and placed in an inappropriately warm room. Sleeping position was not stated in 28 of the SIDS cases but eight were found dead prone and four supine. There was a history of recent upper respiratory infection in 14 cases and of parental drug abuse in three.

Of the nine deaths which presented as suspected homicides, four cases were from proven or strongly suspected deliberate mechanical asphyxia and four were from head injury associated with subdural haemorrhage, three of which involved an element of shaking. The cause of death in the remaining case, a five month old boy, was unascertained but suspected to be from positional asphyxia owing to falling or being thrown head down into a waste paper basket. Table 3 shows the incidence and distribution of petechial haemorrhages in the five suspected asphyxial deaths.

The causes of death among the nine neonates were birth asphyxia (3), birth trauma and lack of attention at birth (2), antepartum haemorrhage, necrotising enterocolitis, aspiration of amniotic fluid, and atrial septal defect. The latter case was subsequently found to have MCAD (see discussion below).

The causes of death among the remaining 14 infants who presented in some other manner were vehicular accidents (3), house fire (2), electrocution, microcephaly, congenital diaphragmatic hernia, accidental asphyxia from entrapment in a broken cot, bathtub drowning, accidental hot water scalds, fall from height, meningococcal meningitis, and an unspecified neurological condition. Owing to family objections, the latter three cases were certified on the basis of external examination and ancillary laboratory investigations, without recourse to necropsy.

Toxicological analyses were negative in 66 cases and positive in 10 cases. The drugs 
Table 2 Incidence of petechial haemorrhages in SIDS and explained "cot deaths"

\begin{tabular}{llllllllll}
\hline & None & External & $\begin{array}{l}\text { Thymus } \\
\text { only }\end{array}$ & $\begin{array}{l}\text { Epicardium } \\
\text { only }\end{array}$ & $\begin{array}{l}\text { Pleura } \\
\text { only }\end{array}$ & $\begin{array}{l}\text { Thymus and } \\
\text { pleura }\end{array}$ & $\begin{array}{l}\text { Epicardium } \\
\text { and pleura }\end{array}$ & $\begin{array}{l}\text { Thymus and } \\
\text { epicardium }\end{array}$ & $\begin{array}{l}\text { All three } \\
\text { sites }\end{array}$ \\
\hline SIDS $(\mathrm{n}=40)$ & 4 & 0 & 2 & 0 & 2 & 6 & 5 & 3 & 18 \\
Explained $(\mathrm{n}=22)$ & 9 & $1^{\star}$ & 1 & 1 & 2 & 3 & 2 & 1 & 2 \\
\hline
\end{tabular}

*7 month old boy who inhaled vomit.

Table 3 Incidence of petechial haemorrhages in deaths from suspected homicidal asphyxia

\begin{tabular}{|c|c|c|c|c|c|}
\hline $\operatorname{Sex}$ & Age & Circumstances & Final cause of death & External petechiae & Internal petechiae \\
\hline Female & 2 months & Suspicious of deliberate asphyxia & Mechanical asphyxia & None & All 3 intrathoracic sites \\
\hline Male & 5 months & $\begin{array}{l}\text { Found head down in waste paper } \\
\text { basket }^{1}\end{array}$ & Unascertained & None & None \\
\hline Male & 10 months & Known deliberate smothering ${ }^{2}$ & Smothering & None & None \\
\hline Male & 29 months & Known deliberate smothering ${ }^{2}$ & Smothering & Tarsal plates & None \\
\hline Male & 28 months & Suspicious of deliberate asphyxia ${ }^{3}$ & $\begin{array}{l}\text { Asphyxia, mechanism } \\
\text { unknown }\end{array}$ & Face & All 3 intrathoracic sites \\
\hline
\end{tabular}

Suspected positional asphyxia.

${ }^{2}$ Deliberate smotherings by father in didactic death.

${ }_{3}^{3}$ Abrasions to lips and nostrils, history of previous episodes of deliberate harm.

detected were those used in resuscitation (three cases), alcohol (three cases of isolated low blood or urinary alcohol, likely artefactual), carbon monoxide (two cases from house fires), carbamazepine (used therapeutically), and dextropropoxyphene (presumably transferred from maternal breast milk). Toxicological analyses were not indicated in 19 cases.

Routine radiological skeletal survey revealed recent skull fractures in two of the infants who died from head injuries and a massive diaphragmatic hernia in a young child presenting with intestinal obstruction. Radiological investigations were negative in all other cases surveyed.

\section{Discussion}

Recent studies on the quantity and quality of perinatal and infant necropsies performed in Wales, Northern Ireland, and northern England have all indicated a failure to achieve the minimum standards proposed by the Royal College of Pathologists. ${ }^{7-9}$ Necropsies on many SIDS cases are performed by forensic or coroner's pathologists primarily to exclude unnatural death and often rank as "poor" by these criteria. $^{8}$

Since SIDS is by definition a diagnosis by exclusion, its incidence in any geographical area will depend on how thoroughly infant deaths are investigated in that locality. Since suggested investigative protocols for infant deaths are typically costly in terms of time and resources, infant necropsies in some geographical areas will inevitably fall short of these ideals, particularly when associated with limited resources and negative expectations. However, experience of the necropsy protocol used in Dundee suggests that such efforts are worthwhile since at least as many infant deaths can be explained by full bacteriological, virological, and biochemical investigations as by necropsy and histological investigation alone. The most productive investigations in this series were bacteriological culture of the CSF and blood, which unexpectedly provided an adequate cause of death in $15 \%$ of apparent cot deaths. These deaths were typically not accompanied by significant gross or histological findings. Routine microbiological investigations also successfully identified the causal organisms in most of the deaths from infective causes which were first suspected during necropsy. Without routine sampling before necropsy detection or confirmation of these cases would not have been possible.

The median time between death and necropsy in this series was five hours. Table 1 shows that even with early necropsy, postmortem contamination or overgrowth by commensals remained a problem. However, significant pathogens were often demonstrated by bacteriological culture of the CSF, blood, and trachea. In contrast, culture of spleen and lung tissue and swabs from the ears, nose, and throat usually yielded insignificant commensals or contaminants. Although postmortem microbiology requires cautious interpretation, the presence of such major pathogens as Streptococcus pneumoniae and Neisseria meningitidis in the $\mathrm{CSF}$ and blood of infants who have died suddenly cannot be dismissed as incidental findings, even when there are no associated naked eye or microscopic meningeal changes. Overwhelming streptococcal septicaemia and meningococcaemia may result in death within a few hours of onset, and intrathoracic petechiae may be the only positive macroscopic findings. ${ }^{10}{ }^{11}$ Similarly, Streptococcus pneumoniae, the commonest pathogen in our series, has a tendency to invade the bloodstream in infancy and early childhood. ${ }^{12}$ If death occurs during this early bacteraemic phase, no meningeal exudate will be apparent at necropsy.

Reported positive diagnostic yields from routine toxicology and radiological skeletal survey are low, with drugs such as antihistamines, antitussives, analgesics, and minor sedatives being detected in $12 \%$ of cases. ${ }^{13}$ Positive toxicological findings, identified in 10 of the 76 cases analysed in this series, were of minor significance and were innocently explained by the history. However, without the reassurance of negative toxicology, deliberate or accidental poisoning as a cause of death cannot be excluded, ${ }^{14}$ and negative results may be of inestimable value if death or injury to a subsequent child occurs at the hands of the same carer or when deliberate poisoning is later suggested, as happened in one of the now infamous Beverley Allitt cases. ${ }^{15}$ 
Biochemical investigations should also be encouraged in all unexplained infant deaths since the detection of a rare inherited metabolic condition will have important consequences for the family. ${ }^{16}$ In the present series one case of medium chain acyl coenzyme-A dehydrogenase (MCAD) deficiency was diagnosed after death in a 23 month old girl who presented as a cot death. This family had also lost their two day old daughter three years previously, the death initially being attributed to an atrial septal defect. However, retrospective biochemical and cytogenetic analysis of frozen blood, left over from routine toxicological analyses, revealed that this neonate was also homozygous recessive for the inherited MCAD deficiency. A third surviving sibling was found to be a heterozygous carrier of the disorder.

Intrathoracic petechial haemorrhages are the most consistent internal finding in SIDS, being present in over $80 \%$ of cases. ${ }^{17}$ Their presence over the thymus, epicardium, and visceral pleura has been taken to be indicative of negative intrathoracic pressures generated by obstructive apnoea and increased inspiratory effort. $^{18}$ Although internal petechiae were present at least one intrathoracic site in $90 \%$ of SIDS cases in this series, their presence in 55\% of explained infant deaths (table 2) supports the suggestion that they may also be a non-specific feature of the final mechanism in many other infant deaths. ${ }^{19}{ }^{20}$ Although the number of cases presented here is small, it appears that the likelihood of finding an adequate cause of death is reduced when internal petechiae are identified at all three intrathoracic sites. The inconsistent presence of petechiae in many cases of known deliberate asphyxia makes it impossible to differentiate these cases from SIDS at necropsy alone, and thorough knowledge of the circumstances is essential. ${ }^{21}$ In the five deliberate asphyxial deaths presented here, internal petechiae were an inconsistent finding and external petechiae were found only in older children (tables 2 and 3 ).

American experience has shown that a thorough death scene investigation often provides an alternative diagnosis to SIDS since many infant deaths can be explained as cases of accidental asphyxia, overlaying, or overheating. ${ }^{22} 23$ This series included the initially suspicious death of a 20 month old girl who had facial and pleural petechiae and linear abrasions across the neck and shoulders. A visit to the scene showed this to be a case of accidental asphyxia caused by entrapment in a broken cot. While co-sleeping of infants with parents remains a common practice, overlaying is an unpopular pathological diagnosis owing to the difficulties in obtaining a clear history and interpreting the non-specific or negative necropsy findings. ${ }^{24}$ Since many of these accidental and asphyxial deaths are preventable, the proposed introduction of an international standard protocol for infant death scene investigation should identify specific risks and further reduce the incidence of SIDS. ${ }^{25}$ It has been suggested that an additional criterion for the categorisation of SIDS should be that "careful death scene investigation by a trained observer reveals no other cause of death," ${ }^{22}$ a view endorsed at the second SIDS global strategy meeting in Norway. $^{2}$

Thanks to increased awareness of modifiable risk factors, such as the prone sleeping position, overheating, and parental smoking, the incidence of SIDS has declined markedly in many countries over recent years. ${ }^{26}$ In the cases which remain, the incidence of concealed deliberate asphyxia will inevitably be increased. The prospects of finding an adequate explanation in apparent cot deaths could be improved by placing more emphasis on the medical history, death scene investigation, and routine laboratory investigations. The data presented here show that the effort involved in performing routine laboratory investigations in all apparent cot deaths is worthwhile in terms of its positive diagnostic yield. In particular, routine bacteriological culture of the CSF and blood, taken by cisternal puncture before necropsy that is ideally started within a few hours of death, should form part of every sudden infant death investigation protocol, whether or not specifically indicated from the history.

I wish to acknowledge the efforts of Dr D E Cox and Professor D J Pounder, who established the necropsy protocol used in Dundee after consultations with The Scottish Cot Death Trust, the Regional Procurator Fiscal, and local departments of paedithe Regional Procurator Fiscal, and local departments of paediatrics, pathology, cytogene
medicine (see appendix 1).

\section{Appendix I}

\section{Paediatric necropsy protocol}

The reporting police officer follows a specific protocol to contact the procurator fiscal and on-call pathologist for an immediate necropsy if the death occurs "out of hours."

CYTOGENETICS

Before necropsy a full thickness skin biopsy from the anterior abdominal wall in $10 \%$ dimethyl sulphoxide (DMSO) for cell culture and cytogenetic examination.

BACTERIOLOGY

Specimens collected under aseptic conditions for routine analysis:

Before necropsy: cerebrospinal fluid by cisternal puncture, subclavian blood by needle puncture.

During necropsy: heart blood or splenic tissue, right lower lobe lung tissue. Other specimens taken where appropriate.

VIROLOGY

Specimens collected under aseptic conditions for routine analysis:

Before necropsy: throat swab in virological transport medium, whole blood for serology.

During necropsy: faeces in sterile universal, ileal contents in sterile universal.

BIOCHEMISTRY

If death occurred within four hours of necropsy:

Before necropsy: bladder urine (frozen) plus serum $(2 \mathrm{ml})$ for biochemical analysis for inborn errors of metabolism. Vitreous humour from one eye for urea analysis.

During necropsy: liver and pons tissue (two $1 \mathrm{~cm}$ cubes of each, frozen to $-60^{\circ} \mathrm{C}$ ) for later biochemical analysis for evidence of storage disease, if considered appropriate.

HISTOLOGY

Histological blocks of epiglottis and larynx (LS), trachea and thyroid, lung (four blocks), thymus, duodenum, ileum, liver (right and left lobes), spleen, mesenteric lymph node, adrenal, kidney, muscle 
(diaphragm and pectoralis minor), costochondral junction (right sixth rib), heart (posterior left ventricle, right ventricle, interventricular septum as $\mathrm{T}$ block) and any others as appropriate.

TOXICOLOGY

Before necropsy: blood for drugs screen and alcohol. Vitreous humour from one eye for alcohol.

During necropsy: liver, stomach contents and urine for drugs screen. Bile or CSF if available.

RADIOGRAPHS

After necropsy: radiographic skeletal survey includes skull $\mathrm{AP}$ and lateral, chest and abdomen, upper and lower limbs.

1 Limerick SR. Sudden infant death in historical perspective. f Clin Pathol 1992;45(suppl):3-6.

2 Byard RW, Becker LE, Berry PJ, et al. The pathological approach to sudden infant death-consensus or confusion? Am F Forensic Med Pathol 1996;17:103-5.

3 Berry PJ, Keeling JW. The investigation of sudden unexpected death in infancy. In: Anthony PP, MacSween RNM, eds. Recent advances in histopathology, vol 14 London: Churchill Livingstone, 1989:251-79.

4 Rajs J. Differential diagnosis of SIDS from the medico-legal point of view. Acta Paediatr Suppl 1993;389:80-1.

5 Royal College of Pathologists. Guidelines for post mortem reports. London: RCP, 1993.

6 Wigglesworth JS, Keeling JW, Rushton DI, et al. Pathological investigations in cases of sudden infant death. $f$ Clin Pathol 1987:41:1481-3.

7 Cartlidge HT, Dawson AT, Stewart JH, et al. Value and quality of perinatal and infant postmortem examinations: cohort analysis of 400 consecutive deaths. BMF 1995;310: 155-8.

8 Thornton CM, O'Hara MD. A regional audit of perinatal and infant necropsies in Northern Ireland. $B r \mathcal{F}$ Obstet and infant necropsies in

9 Wright C, Cameron H, Lamb W. A study of the quality of perinatal autopsy in the former Northern Region. $\mathrm{Br} \mathcal{F}$ Obstet Gynaecol 1998;105:24-8.
10 Wooley PV. Mechanical suffocation during infancy: a comment on its relation to the total problem of sudden comment on its relation to the
death. $\mathcal{F}$ Paediatr $1945 ; 26: 572-5$.

11 Barnham M, Henderson DC. Group B streptococcal infection presenting as sudden death in infancy. Arch Dis Child 1987;62:419-20.

2 Shanson DC. In: Microbiology in clinical practice, 2nd ed. London: John Wright, 1989:236-8.

13 Smialek JE, Monforte JR. Toxicology and sudden infant death. F Forensic Sci 1977;22:757-62.

14 Finkle BS, McCloskey KL, Kopjak L, et al. Toxicological analyses in cases of sudden infant death: a national feasibility study. F Forensic Sci 1979;24:775-89.

15 Berry PJ. The Allitt inquiry-implications for pathologists. Association of Clinical Pathologists News, Summer 1995.

16 Bonham JR, Downing M. Metabolic deficiencies and SIDS. f Clin Pathol 1992;45(suppl):33-8.

17 Kleeman WJ, Wiechern V, Schuck M, et al. Intrathoracic and subconjunctival petechiae in sudden infant death syndrome (SIDS). Forensic Sci Int 1995;72:49-54.

18 Beckwith JB. Intrathoracic petechial haemorrhages: a clue to the mechanism of death in sudden infant death syndrome? Ann NY Acad Sci 1988;533:37-47.

19 Krous HF. The microscopic distribution of intrathoracic petechiae in sudden infant death syndrome. Arch Pathol Lab Med 1984;108:77-9.

20 Krous HF, Jordan J. A necropsy study of distribution of petechiae in non-sudden infant death syndrome. Arch petechiae in non-sudden infa
Pathol Lab Med 1984;108:75-6.

21 Sadler DW. Two cases of homicidal strangulation first discovered at autopsy. F Clin Pathol 1994;47:679-80.

22 Bass M, Kravath RE, Glass L. Death scene investigation in sudden infant death. N Engl f Med 1986;315:100-5.

23 Gilbert-Barnes E, Hegstrand L, Chandra S, et al. Hazards of mattresses, beds and bedding in deaths of infants. $A m \mathcal{F}$ Forensic Med Pathol 1991;12:27-32.

24 Byard, RW. Hazardous infant and early childhood sleeping environments and

25 Iyasu S, Hanzlik R, Rowley D, et al. Proceedings of "Workshop on guidelines for investigation of sudden unexplained infant deaths", July 12-13, 1993. F Forensic Sci 1994;39: infant death.

26 Gibson AAM. Current epidemiology of SIDS. 7 Clin Pathol 1992;45(suppl):7-10. 